\title{
LETTERS
}

\section{No evidence of increased risk of acquired rifampin resistance}

We read with interest the recent CMAJ commentary by Batt and Khan ${ }^{1}$ about our recent publications of trials comparing 4 months of rifampin with 9 months of isoniazid in adults ${ }^{2}$ and children for latent tuberculosis (TB) infection. ${ }^{3}$ In those 2 trials, and in an earlier trial involving adults, ${ }^{4}$ we have shown that 4 months of rifampin is consistently significantly superior to 9 months of isoniazid for completion and safety in adults and is very safe and well tolerated in children for treatment of latent TB. In both populations, the efficacy of 4 months of rifampin was noninferior to that of 9 months of isoniazid. ${ }^{2,3}$ In summary, 4 months of rifampin is shorter, safer, better-completed and as effective as 9 months of isoniazid. Seems like an easy choice for patients and providers.

However, Batt and Khan raise concerns regarding the potential risk of creating rifampin resistance if 4 months of rifampin is given to patients with undetected active TB. ${ }^{1}$ We agree that creation of resistance by inadvertent monotherapy of active TB is a very important potential risk, but we believe it is important to adequately review the available evidence regarding this risk. There have been 2 relevant systematic reviews. ${ }^{5,6}$ The first, cited by Batt and Khan, reviewed 6 studies of regimens containing rifamycin and found no evidence of increased risk of acquired rifampin resistance. ${ }^{5}$ The second reviewed 13 studies, including 6 involving people infected with HIV, and found no evidence of increased risk of acquired resistance to isoniazid in randomized trials or cohort studies using isoniazid to treat latent TB. ${ }^{6}$ This evidence suggests that risk of acquired drug resistance from latent TB therapy is very low.

Batt and Khan recommend that clinicians in settings without access to sputum induction refer selected patients (e.g., those at high risk of active TB, or with clinical or radiographic signs of active TB) who are unable to provide sputum samples spontaneously to centres where this is performed. What is the evidence that sputum induction is necessary? To answer this, we reviewed carefully the methods used to exclude active TB before initiating treatment for latent TB infection in all the published studies included in the 2 systematic reviews. 5,6 Our reasoning was that, since there was no evidence of acquired resistance, the methods to exclude active TB in these studies were adequate to safeguard against creating resistance. Of the 17 studies, all but 2 described the procedures used to exclude active TB before starting latent TB therapy; none of these 15 studies described use of sputum induction to exclude active TB before starting latent TB therapy. The evidence from all these studies suggests that symptom assessment, physical examination, chest radiography and spontaneous sputum collection is adequate to prevent the emergence of drug resistance. We suggest that Batt and Khan should provide supportive evidence for their opinion that sputum induction is essential. This procedure is inaccessible in many settings and, if essential, would create a barrier to safe preventive therapy for many populations.

The authors also suggest that creation of isoniazid resistance is preferable to the development of rifampin resistance. However, there is published evidence that treatment outcomes in patients with isoniazid-resistant strains are significantly worse, including frequent progression to multidrug-resistant TB. ${ }^{7}$ Batt and Khan cite the difficulties of treating rifampin-resistant $T B$, with lengthy regimens including many months of injections, and ignore recommendations made in 2018 by the World Health Organization for an all-oral regimen as first-line treatment of rifampinresistant $\mathrm{TB},{ }^{8}$ which uses much more effective drugs. ${ }^{9}$

Finally, the authors suggest that the study population does not reflect the typical Canadian clinical experience. In the
4 months of rifampin trials we have conducted, a total of 1748 participants were enrolled at Canadian sites. ${ }^{2-4}$ This is, we believe, the largest number of participants in any randomized trial of latent TB therapy enrolled in Canada. If these results are not relevant to Canadian practice, what would be?

In summary, while we acknowledge that the concerns regarding rifampin resistance are important, we believe there is a substantial body of evidence that symptom screening, physical examination, chest radiography and spontaneous sputum examination will be adequate to safeguard against this risk. We believe that 4 months of rifampin offers very important benefits for patients in Canadian settings, as it is a better tolerated, more acceptable and much safer regimen for latent TB therapy than the current standard of 9 months of isoniazid.

\section{Dick Menzies MD MSc}

Professor, Department of Epidemiology, Biostatistics and Occupational Health, McGill University, Montréal, Que.

\section{Victoria Cook MD}

Clinical associate professor, Department of Medicine, University of British Columbia, Vancouver, BC

\section{Richard Long MD}

Professor, Department of Medicine, Faculty of Medicine and Dentistry, University of Alberta, Edmonton, Alta.

\section{Rovina Ruslami MD PhD}

Professor, Department of Biomedical Sciences, Division of Pharmacology \& Therapy, Faculty of Medicine, Universitas Padjadjaran, Bandung, Indonesia

Cite as: CMAJ 2019 November 25;191: E1314-5. doi: 10.1503/cmaj.73353

\section{References}

1. Batt J, Khan K. Responsible use of rifampin for the treatment of latent tuberculosis infection. CMAJ 2019;191:E678-9.

2. Menzies D, Adjobimey M, Ruslami R, et al. Four months of rifampin or nine months of isoniazid for latent tuberculosis in adults. N Engl J Med 2018;379:440-53. 
3. Diallo T, Adjobimey M, Ruslami R, et al. Safety and side effects of rifampin versus isoniazid in children. N Engl J Med 2018;379:454-63.

4. Menzies D, Long R, Trajman A, et al. Adverse events with 4 months rifampin or 9 months isoniazid as therapy for latent TB infection: results of a randomized trial. Ann Intern Med 2008;149:689-97.

5. den Boon S, Matteelli A, Getahun H. Rifampicin resistance after treatment for latent tuberculous infection: a systematic review and meta-analysis. Int J Tuberc Lung Dis 2016;20:1065-71.

6. Balcells ME, Thomas S, Godfrey-Faussett P, et al. Isoniazid preventive therapy and risk for resistant tuberculosis. Emerg Infect Dis 2006;12:744-51.

7. Gegia M, Winters N, Benedetti A, et al. Treatment of isoniazid-resistant tuberculosis with first-line drugs: a systematic review and meta-analysis. Lancet Infect Dis 2017;17:223-34.

8. WHO consolidated guidelines on drug-resistant tuberculosis treatment. Geneva: World Health Organization; 2019.

9. Collaborative Group for the Meta-Analysis of Individual Patient Data in MDR-TB treatment - 2017; Ahmad N, Ahuja SD, Akkerman OW, et al. Treatment correlates of successful outcomes in pulmonary multidrug-resistant tuberculosis: an individual patient data meta-analysis. Lancet 2018;392:821-34.

Competing interests: None declared. 